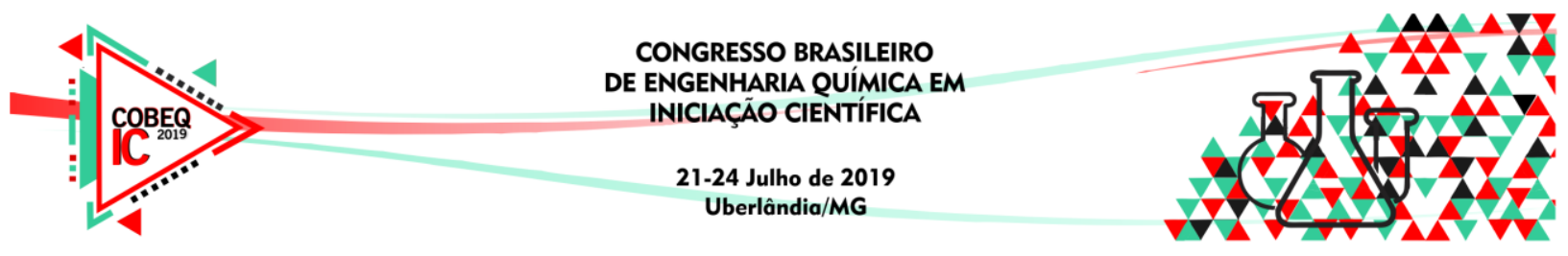

\title{
Otimização da recuperação de compostos antioxidantes da casca de banana
}

\author{
L. OLIVEIRA ${ }^{1,2}$, E. S. FIGUEIREDO ${ }^{2}$, E. P. JUNG ${ }^{2}$, C. N. KUNIGAMI ${ }^{2}$, L. O. RIBEIRO ${ }^{2}$ \\ ${ }^{1}$ Universidade Federal Fluminense, Faculdade de Engenharia Química \\ ${ }^{2}$ Instituto Nacional de Tecnologia, Divisão de Química Analítica \\ E-mail para contato: lucas.oliveira@int.gov.br
}

\begin{abstract}
RESUMO - As condições climáticas como temperatura, umidade relativa, precipitação e insolação permitem que a bananicultura seja distribuída durante todo o ano e difundida em diversos estados brasileiros, atendendo, de forma regular, às necessidades de consumo. Porém, a industrialização expressiva da fruta gera um passivo ambiental importante, representado pelas cascas. Estas representam cerca de $30 \%$ da massa total da fruta madura. Entretanto, este resíduo abundante é rico em compostos de alto valor agregado como os compostos fenólicos, antioxidantes naturais, mostrando que o aproveitamento do mesmo é uma alternativa de grande importância econômica. Portanto, o objetivo deste trabalho foi otimizar a extração destes compostos, utilizando um planejamento experimental, no qual o percentual de etanol na solução extratora e a razão sólido/líquido foram as variáveis independentes e a concentração de compostos fenólicos e a capacidade antioxidante, as resposta ao delineamento. Os resultados mostraram que os efeitos quadráticos e lineares do percentual de etanol na solução extratora foram os responsáveis pela recuperação dos compostos fenólicos e, consequente aumento na capacidade antioxidante dos extratos. Usando a ferramenta desejabilidade, observou-se que a melhor condição operacional para a obtenção de um extrato fonte de compostos fenólicos e com elevada capacidade antioxidante é alcançada quando a farinha é extraída usando etanol $42 \%$ em água e razão sólido/líquido de 1:33.
\end{abstract}

Palavras-chave: Farinha da casca de banana. Extração sólido-líquido. Capacidade antioxidante. Compostos fenólicos.

\section{INTRODUÇÃO}

De acordo com a Organização das Nações Unidas para a Alimentação e a Agricultura (2018), a produção mundial de banana em 2017 foi cerca de 114 milhões de toneladas, sendo os principais produtores Índia, China, Indonésia, Brasil, Equador, Filipinas. A produção brasileira, em 2018, foi cerca de 7,23 milhões de toneladas.

A banana é a segunda fruta mais consumida mundialmente, e a primeira no Brasil (ANUÁRIO, 2015). A produção e o consumo expressivo da banana geram um passivo ambiental importante, correspondente à casca das frutas, a qual representa cerca de $30 \%$ da massa total da fruta madura. 

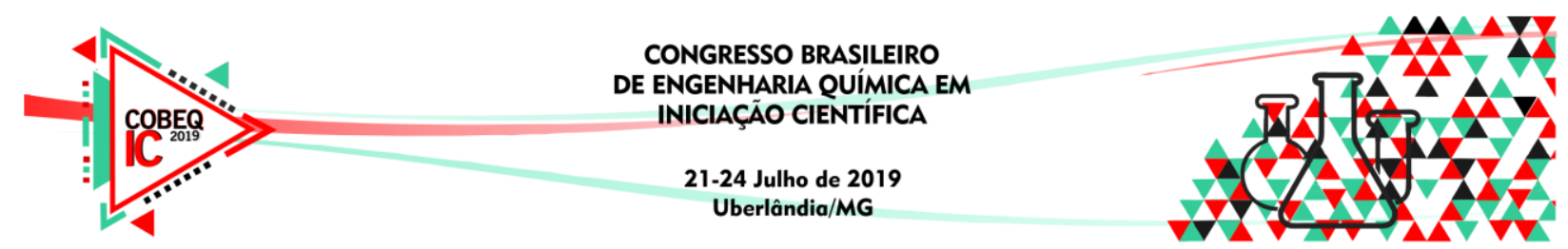

Rebelo et al. (2014), ao avaliarem a composição fenólica de uma farinha de casca de banana (Musa AAA), detectaram a presença de flavonoides, destacando os flavan-3-óis e flavonóis. Além dos monômeros (+)-catequina, (-)-epicatequina e (-)-galocatequina. Estes compostos exibem inúmeros efeitos biológicos, incluindo ação antioxidante e antiviral (BRUM., 2006).

Compostos antioxidantes, como os fenólicos, são capazes de neutralizar e/ou desestabilizar parcialmente os radicais livres, reduzindo a oxidação do LDL (Low Density Lipoprotein), protegendo o organismo de doença arterial coronariana, câncer e doenças neurodegenerativas (SAURA-CALIXTO;GONI, 2006). Estes autores, ao avaliarem a capacidade antioxidante dos principais alimentos que compõem a dieta mediterrânea, destacaram que bebidas como café, chá, vinho e suco de laranja e o consumo de frutas foram aqueles que mais contribuíram para a capacidade antioxidante desta dieta. Deste modo, o extrato da farinha da casca de banana pode incrementar os níveis de compostos antioxidantes nos alimentos, além de contribuir para sua estabilidade frente ao armazenamento.

Tendo em vista os indícios de problemas que podem ser provocados pelo consumo de antioxidantes sintéticos, pesquisas têm sido conduzidas no sentido de encontrar produtos naturais com ação antioxidante, visando à substituição destes em produtos alimentícios e cosméticos (RAMALHO; JORGE, 2006).

Assim, o objetivo deste trabalho foi avaliar os extratos, quanto à capacidade antioxidante por meio do método ABTS e a concentração de compostos fenólicos totais, visando obter-se a condição otimizada.

\section{DESENVOLVIMENTO}

Para realização deste estudo foram utilizadas cascas de bananas da variedade nanica (Musa cavendishi), popularmente conhecida como banana d'água, doadas por uma empresa produtora de doce de banana localizada no Estado do Rio de Janeiro.

Tabela 1 - Planejamento experimental do tipo composto central rotacional para recuperação dos compostos bioativos da farinha da casca de banana: variáveis independentes e dependentes

\begin{tabular}{|c|c|c|c|c|c|c|}
\hline \multirow{3}{*}{ Tratamentos } & \multicolumn{2}{|c|}{ Variáveis codificadas } & \multicolumn{2}{c|}{ Variáveis reais } & \multicolumn{2}{c|}{ Resultados } \\
\cline { 2 - 7 } & $\begin{array}{c}\text { Razão } \\
\text { sólido:líquido }\end{array}$ & $\begin{array}{c}\% \\
\text { Etanol }\end{array}$ & $\begin{array}{c}\text { Razão } \\
\text { sólido:líquido }\end{array}$ & $\begin{array}{c}\% \\
\text { Etanol }\end{array}$ & $\begin{array}{c}\text { CFT } \\
(\mathrm{mg} / 100 \mathrm{~g})\end{array}$ & $\begin{array}{c}\text { ABTS } \\
(\mu \mathrm{mol} \text { Trolox/g) }\end{array}$ \\
\hline 1 & +1 & 1 & 30 & 85 & 380,42 & 13,12 \\
\hline 2 & -1 & 1 & 13 & 85 & 365,14 & 11,40 \\
\hline 3 & +1 & -1 & 30 & 15 & 460,09 & 18,94 \\
\hline 4 & -1 & -1 & 13 & 15 & 418,53 & 16,40 \\
\hline 5 & $+1,41$ & 0 & 33 & 50 & 540,27 & 26,42 \\
\hline 6 & $-1,41$ & 0 & 10 & 50 & 648,44 & 21,08 \\
\hline 7 & 0 & 1,41 & 22 & 99 & 382,34 & 7,87 \\
\hline 8 & 0 & $-1,41$ & 22 & 1 & 454,94 & 13,24 \\
\hline 9 & 0 & 0 & 22 & 50 & 505,25 & 24,46 \\
\hline 10 & 0 & 0 & 22 & 50 & 512,64 & 24,17 \\
\hline 11 & 0 & 0 & 22 & 50 & 495,39 & 22,05 \\
\hline
\end{tabular}




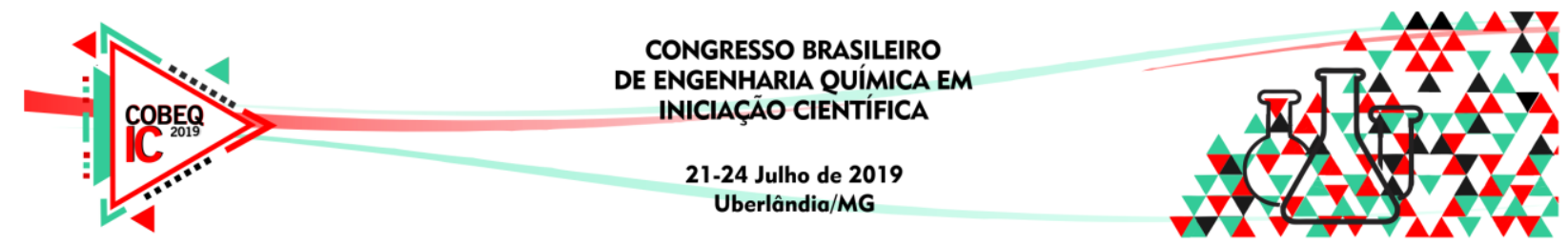

As cascas foram transportadas até o Instituto Nacional de Tecnologia sob refrigeração. Após o recebimento, as cascas foram secas a $50{ }^{\circ} \mathrm{C}$ por $48 \mathrm{~h}$ para elaboração de uma farinha, a partir de seu trituramento com um mixer portátil. Com a farinha obtida foi realizado o processo de extração sólido-líquido a $60^{\circ} \mathrm{C}$ sob agitação magnética $(500 \mathrm{rpm})$ por 30 minutos, variando a concentração de etanol na solução extratora e a razão sólido-líquido por meio de um delineamento experimental do tipo composto central rotacional conforme mostra a Tabela 1. O extrato foi filtrado e, posteriormente armazenado sob congelamento até a realização dos ensaios de compostos fenólicos totais (CFT) e capacidade antioxidante pelo método ABTS.

\subsection{Métodos analíticos}

A determinação dos compostos fenólicos totais dos extratos foi realizada de acordo com o método proposto por SINGLETON \& ROSSI. (1965), usando o reagente de FolinCiocalteau. Os resultados foram expressos em $\mathrm{mg}$ ácido gálico/100 g. A capacidade antioxidante foi determinada pelo método ABTS. Neste caso, os resultados foram expressos em $\mu$ mol de trolox/g (GIÃO et al., 2007).

\section{RESULTADOS E DISCUSSÃO}

\subsection{ABTS}

Quanto aos resultados da capacidade antioxidante medida pelo método ABTS, ressaltase que eles estão em acordo com aqueles reportados por ORTIZ (2016), que ao avaliar os extratos de casca de banana utilizando uma extração sequencial usando como solventes éter, etanol e água, ambos com razão 1:20 (m/v), obteve-se 20,87 $\mu$ mol Trolox/g. Este valor se encontra na faixa observada em nosso estudo, a qual variou de 7,87 a 26,42 $\mu$ mol Trolox/g, como pode ser visto na Tabela 1.

\subsection{Compostos Fenólicos}

A faixa de compostos fenólicos observada no presente trabalho $(365-648 \mathrm{mg} / 100 \mathrm{~g})$ está em acordo com os resultados reportados por Fatemeh et al. (2012). Estes autores encontraram $585 \mathrm{mg} / 100 \mathrm{~g}$ de compostos fenólicos totais no extrato de farinha de casca de banana, variedade Cavendish, usando metanol $80 \%$ como solução extratora e razão sólidolíquido de 1:20.

\subsection{Influência das Variáveis Independentes}

Com relação às variáveis operacionais, verifica-se por meio da Figura 1 que a concentração de etanol na solução extratora foi a variável com maior influência para a recuperação dos compostos fenólicos e aumento da capacidade antioxidante. Tanto o efeito linear (L) quanto o quadrático (Q) desta variável foram significativos. O efeito quadrático da concentração de etanol apresentou valor negativo. Deste modo, o aumento do percentual de etanol na solução extratora é limitado por um plateau, tendo comportamento inversamente proporcional à capacidade antioxidante do extrato e a recuperação dos compostos fenólicos. 


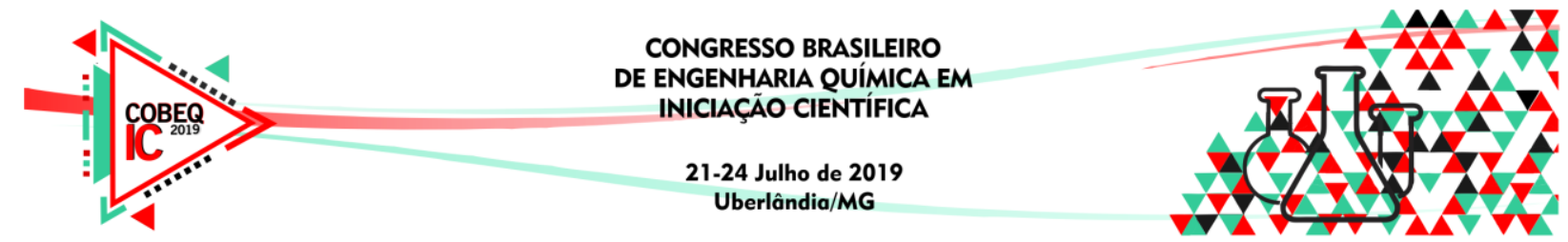

Figura 1 - Influência das variáveis independentes na recuperação de compostos fenólicos (A) e na capacidade antioxidante (B) da farinha da casca de banana
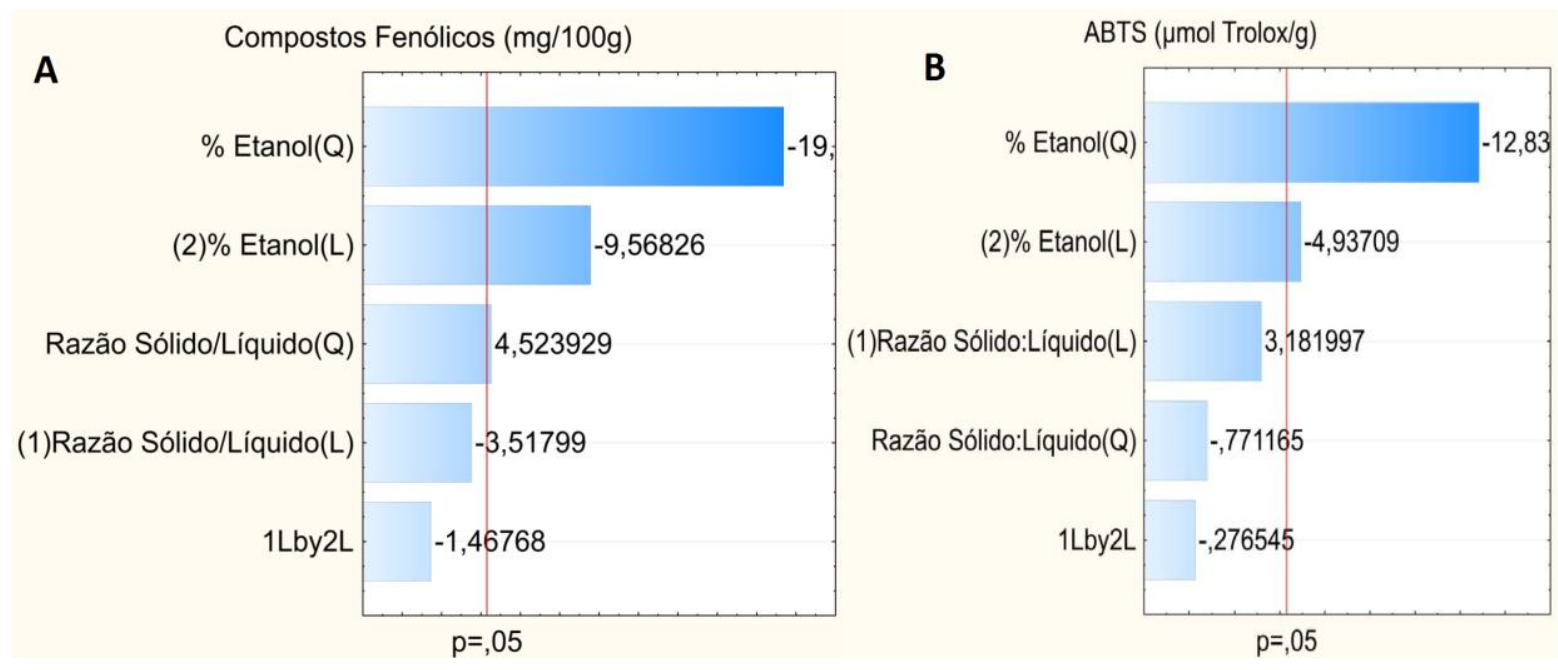

\subsection{Otimização}

A Figura 2 mostra como o valor de desejabilidade (função de otimização) varia em função das condições operacionais, por meio da avaliação estatística dos resultados obtidos para o teor de compostos fenólicos totais e capacidade antioxidante. Deste modo, a zona em vermelho mais intenso (circulada em azul) compreende as condições com melhores resultados para as respostas avaliadas, na qual a concentração de etanol na solução extratora variou de $35 \%$ a 50\% e a razão sólido/líquido de 1:30 a 1:34.

Figura 2 - Superfície de contorno das variáveis independentes em função da desejabilidade.

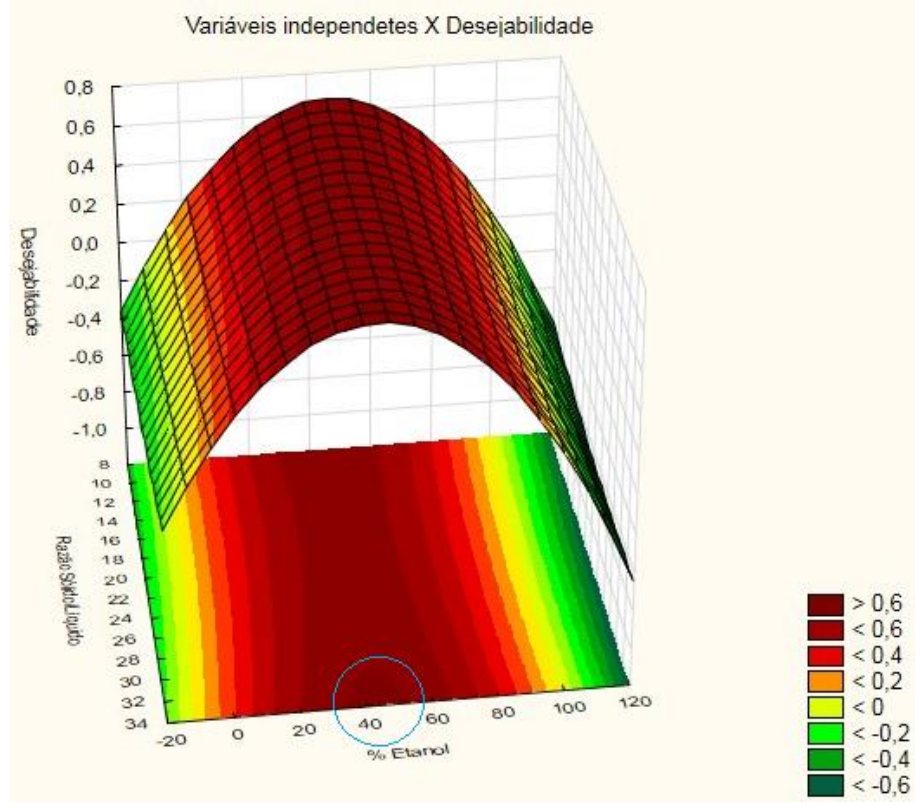




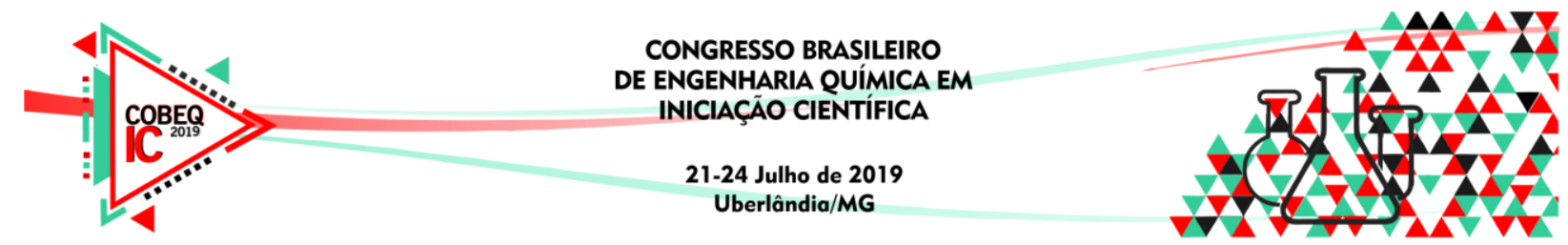

Ao analisar os resultados utilizando a ferramenta desejabilidade em função das respostas obtidas pelo planejamento experimental, levando em consideração os 11 tratamentos e toda a faixa abrangida pelo mesmo, a melhor condição para extração de compostos fenólicos ocorre quando uma solução extratora com $42 \%$ de etanol e uma razão sólido/líquido de 1:33 são utilizadas. $\mathrm{O}$ valor de $\mathrm{D}$ (desejabilidade), que varia de 0 a 1 , foi igual a 0,73 nesta condição operacional, sugerindo bom ajuste dos dados ao modelo estatístico proposto, como pode ser observado na Figura 3.

Figura 3 - Otimização das variáveis para recuperação dos compostos antioxidantes da farinha da casca de banana

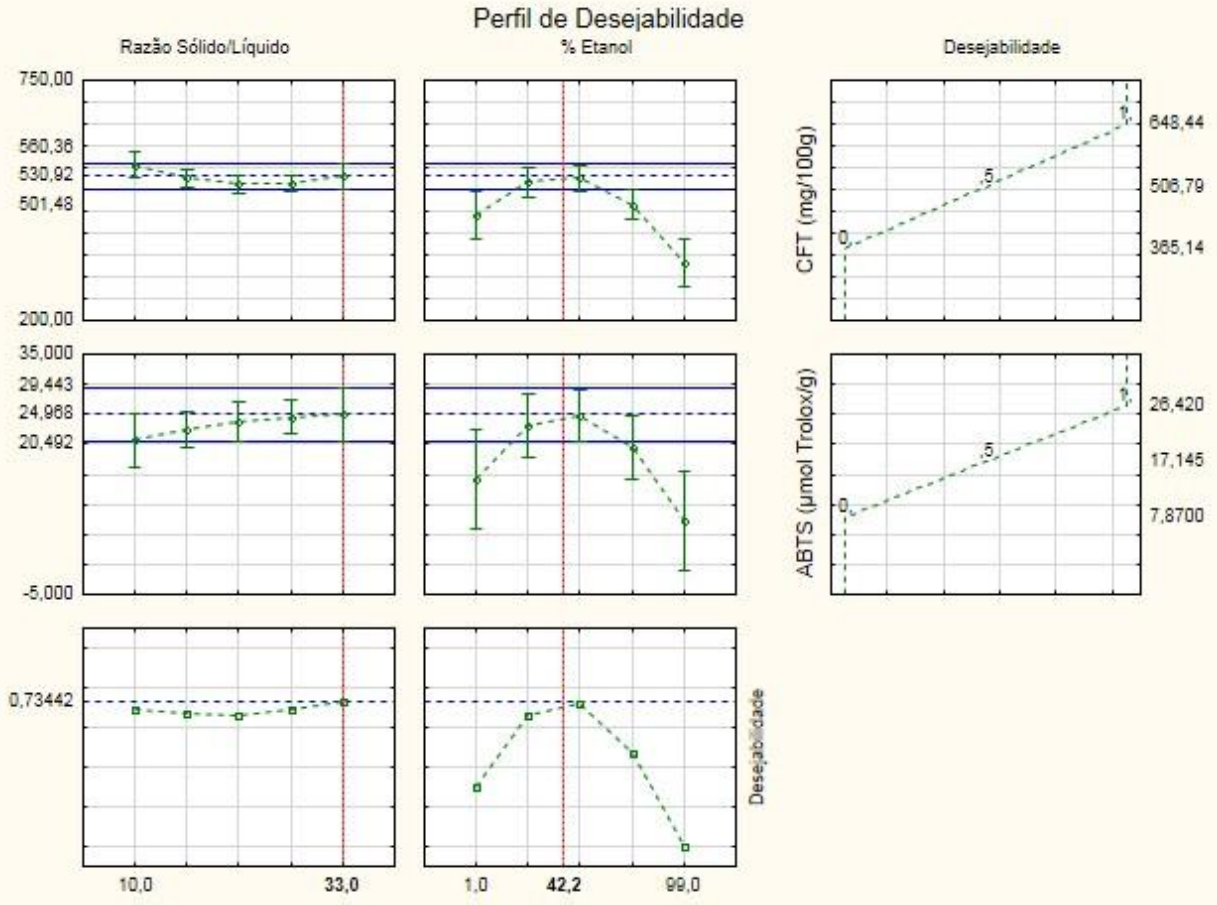

\section{CONCLUSÃO}

Por meio dos resultados obtidos foi possível identificar a melhor condição de extração dos compostos antioxidantes da farinha da casca de banana que, segundo a função desejabilidade, foi aquela na qual se utilizou uma solução extratora com $42 \%$ de etanol e uma razão sólido/líquido de 1:33.

Os resultados obtidos neste estudo demonstram o potencial do resíduo para a obtenção de um extrato fonte de compostos antioxidantes, o qual pode ser empregado em formulações alimentícias, uma vez que se trata de extratos obtidos por meio de um solvente verde, o etanol. 


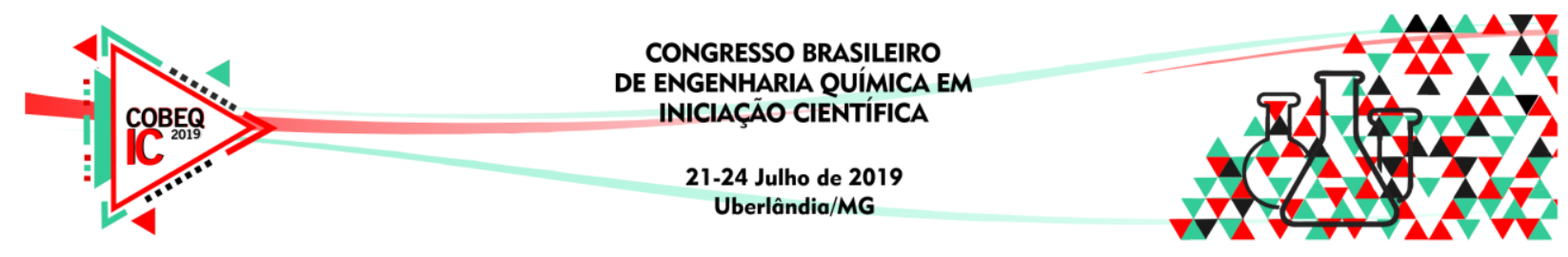

\section{REFERÊNCIAS}

Anuário brasileiro da fruticultura. Ed Gazeta Santa Cruz, Santa Cruz do Sul. 2015.

BRUM, L. P. Atividade antiviral dos compostos fenólicos (ácidos ferúlico e transcinâmico) e dos flavonoides (quercetina e kaempherol ) sobre os herpesvirus bovino 1 , herpesvírus bovino 5 e vírus da cinomose canina. Tese de doutorado. 2006.

CALIXTO-SAURA, F.; GOÑI, I. (2004) Antioxidant capacity of the Spanish Mediterranean diet. Food Chemistry, 94, p.442-447. (2006).

FATEMEH, S. R.; SAIFULLAH, R.; ABBAS, F. M. A; AZHAR, M. E. Total phenolics, flavonoids and antioxidant activity of banana pulp and peel flours: influence of variety and stage of ripeness. International Food Research Journal, 19, p.1041-1046. 2012.

GIÃO, M.S.; GONZÁLES-SANJOSÉ, M.L.; RIVERO-PÉREZ， M.D.; PEREIRA， C.I.; PINTADO, M.E.; MALCATA, F.X. Infusions of Portuguese medicinal plants: Dependence of final antioxidant capacity and phenol content on extraction. Journal of the Science of Food and agriculture, 87, 2638-2647. (2007).

ORTIZ, W.D.; CASCAS DE FRUTAS: ESTUDO DAS PROPRIEDADES NUTRICIONAIS E TECNOLÓGICAS. Dissertação de Pós-Graduação. 2016.

RAMALHO, V. C.; JORGE, N. Antioxidantes utilizados em óleos, gorduras e alimentos gordurosos. Quimica Nova, 29, p.755-760. 2006.

R, L. P. G.; RAMOS, A. M.; PERTUZATTI, P. B.; BARCIA, M. T.; CASTILLO-MUÑOZ, N.; HERMOSÍN-GUTIÉRREZ, I. Flour of banana (Musa AAA) peel as a source of antioxidant phenolic compounds. Food Research International, 55, p.397-403. 2014.

SINGLETON, V.L.; ROSSI, J.A. Colorimetry of total phenolics with phophomolybidicphosphotungstic acid reagents. Am J. Enol. Vitic., 16, p.144. 1965. 